\title{
Anosmia as The Only Symptom of Meningioma
}

\section{Dovile Stankevice* | Therese Ovesen}

*Correspondence: Dovile Stankevice

Address: ENT department, Regional Hospital West Jutland, Laegaardvej 12, DK-7500 Holstebro, Denmark

e-mail $\bowtie:$ dovsta@rm.dk

Received: 26 November 2020; Accepted: 01 December 2020

Copyright: (C) 2020 Stankevice D. This is an open-access article distributed under the terms of the Creative Commons Attribution License, which permits unrestricted use, distribution, and reproduction in any medium, provided that the original work is properly cited.

\section{ABSTRACT}

Dysfunctional sense of smell is frequent. In one in four cases, the cause of anosmia can not be identified. We present a patient with a large olfactory meningioma as the cause of idiopathic anosmia. The patient had no other symptoms of intracranial tumor at all. The medical history emphasizes the importance of MRI scan of the brain in the examination of possible idiopathic anosmia. Furthermore, we describe assessment and further examination of idiopathic anosmia.

Keywords: Anosmia, Olfaction, Meningioma

\section{Introduction}

Dysfunctional sense of smell is frequent. Thus, 15\% complain of decreased sense of smell (hyposmia), while anosmia (loss of sense of smell) is experienced by $1 \%$ of the population1. Several etiological factors such as sinonasal diseases, head traumas, viral respiratory infections, iatrogenic and congenital mechanisms are known causes of anosmia. In approximately one of four cases, the anosmia is idiopathic, as potential triggers cannot be identified (Fjaeldstad et al., 2020). In these cases, MRI of the brain (MRIc) may be performed in order to exclude cerebral pathology.

We present a medical history, where olfactory meningioma was the cause of idiopathic monosymptomatic anosmia.

\section{Case Report}

A 55-year-old woman was referred to the Flavour Clinic at the ENT department, Region Hospital West Jutland, due to monosymptomatic anosmia for six years. The anosmia may have occurred in relation to a viral upper respiratory tract infection. At the beginning of symptoms, the patient had consulted a practicing ENT specialist, who prescribed prednisolone injection. Because of lack of effect, the patient consulted a biopath in vain. In addition to the anosmia, the patient had short phantosmia episodes, i.e olfactory experience without external source (phantom sensing). Prior to referral to the 
Flavour Clinic, the patient had attempted saline rinsing of the nose and nasal steroid without improving the sense of smell. She had no complaints of nasal secretion, nasal stenosis or upper respiratory allergy.

Objectively, ENT examination was normal incl. nasal endoscopy of the olfactory cleft on both sides; normal otoneurologic examination and no pathology in the oral cavity or pharynx. Cutaneous allergy test was negative. CT of the sinuses was normal with fully pneumatized sinus system.

The patient completed the following questionnaires: Sino-Nasal Outcome Test 22: 7 (normal); Major Depression Inventory: 5 (normal); Mini-Mental State Examination: 29 (normal). In addition, Flavour questionnaire about age, gender, medical history, medication, tobacco, alcohol status, etc.

The sense of smell was assessed with extended "Sniffin 'Sticks" test with threshold (T), discrimination (D), identification (I): TDI score. The patient's overall TDI score was 14.75, which confirmed the patient's subjective sensation of anosmia. Basic taste screening was normal.

On suspicion of possible post-infectious mechanism, the patient was recommended olfactory training for 6 months. At follow-up, the anosmia was unchanged, and 1.5 Tesla MRIc was performed, which showed a 3.1x 3x $3 \mathrm{~cm}$ olfactory groove meningioma involving both olfactory bulbs (Fig. 1). The patient was referred for neurosurgical examination and was recommended clinical follow-up and possibly surgical treatment later.

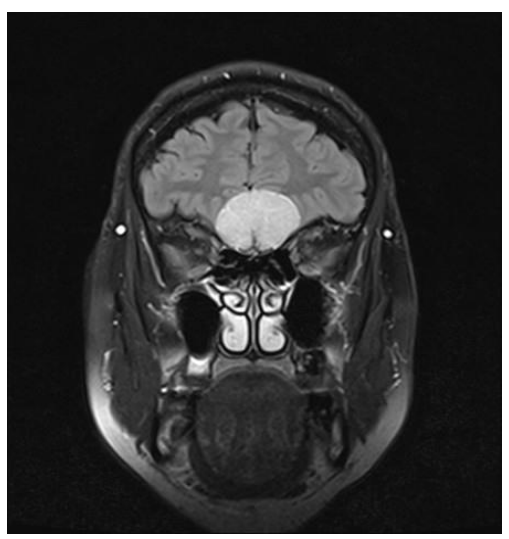

Figure 1: Coronal MRIc of patient's olfactory meningioma

\section{Discussion}

The etiology of anosmia can in most cases be identified on the basis of patient history, objective ENT examination, including rhinoscopy, smell and taste assessment, allergy screening and CT of the sinuses. Further examination of suspected idiopathic anosmia includes 1.5 Tesla MRc for exclusion of central pathology, especially intracranial neoplasms of the anterior cerebral fossa. In the literature, 
intracranial neoplasia is the cause of anosmia in $0.3-1.3 \%$ of cases hey ko (Hoekman et al., 2014; Higgins and Lane, 2014; Decker et al., 2013). However, 40\% of MRc show benign pathology unrelated to anosmia, especially white matter lesions (Decker et al., 2013).

About $10 \%$ of all meningiomas are olfactory groove meningiomas, and most often present with affected sense of smell, headache, visual disturbances, and change in mental status (Adappa et al., 2011). Meningioma is the most common, slow-growing, benign intracranial tumor. The prevalence for women / men is 2: 1, and the incidence increases with age. Diagnosis and symptomatology depend on localization as well as tumor size, as pressure on nearby structures results in typical symptoms: personality change, aphasia, headache, epilepsy, and specific cranial nerve failure. The diagnosis is obtained by MRIc, and in 15\% of olfactory meningioma cases, the suspicion is raised by finding a pale grayish unilateral tumor during nasal endoscopy. Treatment of symptomatic olfactory meningioma is surgical resection with or without endoscopic endonasal assistance, which however will not restore the sense of smell.

\section{Conclusion}

The presented case emphasizes the importance of MRc in the work up of idiopathic anosmia. However, routine MRc is often not recommended up front due to irrelevant findings of non-significant pathology in $1 / 3$ of the patients. We strongly recommend MRc for concomitant neurological / mental / cognitive symptoms, and in idiopathic anosmia cases without improvement of rehabilitation with olfactory training.

\section{References}

Adappa ND, Lee JYK, Chiu AG, Palmer JN. Olfactory Groove Meningioma. Otolaryngol Clin North Am 2011; 44: 965-980.

Decker JR, Meen EK, Kern RC, Chandra RK. Cost effectiveness of magnetic resonance imaging in the workup of the dysosmia patient. Int Forum Allergy Rhinol 2013; 3: 56-61.

Fjaeldstad A, Stankovic J, Onat M, Stankevice D, Ovesen T. Patients and experiences from the first danish flavour clinic. Dan Med J 2020; 67(4).

Higgins TS and Lane AP. What is hey the best imaging modality to investigate olfactory dysfunction in the setting of normal endoscopy? Laryngoscope 2014; 124: 4-5.

Hoekman PK, Houlton JJ, Seiden AM. The utility of magnetic resonance imaging in the diagnostic evaluation of idiopathic olfactory loss. Laryngoscope 2014; 124: 365-368. 